Journal of Case Reports 2017;7(3):322-324

\title{
Pregnancy with Meningioma: Report of Two Rare Cases
}

\author{
Anoosha K Ravi, Poonam Sachdeva, Gauri Gandhi, Anubhuti Rana \\ Department of Obstetrics and Gynaecology, Maulana Azad Medical College, New Delhi, India.
}

\section{Corresponding Author: \\ Dr. Anoosha K Ravi \\ Email: anoosharavi4@gmail.com}

This is an Open Access article distributed under the terms of the Creative Commons Attribution License (creativecommons.org/ licenses/by/3.0).

Received Accepted

Published

May 7, 2017

August 19, 2017

September 30, 2017

\begin{abstract}
Background: Meningioma in pregnancy is a rare occurrence. Whenever it is accompanied with pregnancy, it is mostly symptomatic with complaints like headache, vomiting, visual disturbance, seizures or altered sensorium. The management is challenging as the fetal well-being is also affected along with complications of raised intracranial pressure during labour. Case Report: Here we discuss two such cases of meningioma in third trimester. One was frontal meningioma with intact neurological functions. On the other hand, second patient had a left sphenoid meningioma with left optic atrophy and right hemiparesis. Both of them underwent cesarean delivery under general anesthesia. However, the neurosurgical excision of meningioma was accompanied with the delivery in the second case as the situation demanded the same. Conclusion: These cases always warrant a multimodality approach involving obstetrician, neonatologist, anaesthetist and neurosurgeon. The timing of delivery or neurosurgery depends on the clinical situation like fetal maturity, neurological condition, progress of symptoms and patients' wishes.
\end{abstract}

Keywords: Headache, Meningeal Neoplasms, Meningioma, Neurosurgery, Pregnancy.

\section{Introduction}

Meningioma in pregnancy can present with wide range of symptoms from isolated seizures to deteriorating neurological condition. Prioritizing the fetal maturity or the neurological condition is a challenge. This and other aspects of managing a case of meningioma are explained with the following two cases.

\section{Case Report}

First case was a 32 year old primigravida with left frontal meningioma with history of focal seizures. Patient presented to the antenatal clinic with 36 weeks gestation with well-preserved neurological functions. It was a $2.5 \times 4.6 \mathrm{~cm}$ extraaxial dural based lesion in left frontal region with moderate peri-lesional edema with $3 \mathrm{~mm}$ midline shift on MRI of brain. She was already on tablet levetiracetam and tablet dexamethasone. As the condition of both the patient and the fetus was not compromised, it was decided after consultation along with neurosurgeon to prolong pregnancy until elective cesarean delivery at 37 weeks. At 36 weeks 5 days gestation, she went into spontaneous labour and cesarean section was done under general anaesthesia with rapid sequence intubation. A $2.8 \mathrm{~kg}$ healthy baby was delivered. There was an uneventful post-operative recovery. She was discharged on tablet dexamethasone and levetiracetam until she awaited neurosurgery. She underwent craniotomy a month later and on 2 years follow up she is healthy with no complaints.

Second case was a 22 year old primigravida with 8 months amenorrhea. She presented with history of blindness in left eye and generalized tonic clonic seizures followed by loss of speech. On MRI of brain, an extra-axial dural based mass lesion in left greater wing of sphenoid of about 4 $\times 4 \mathrm{~cm}$ with peri-lesional edema with midline shift of $8.5 \mathrm{~mm}$ and sub-acute infarct was seen. On examination patient was conscious with a Glasgow Coma Scale of 14 with right sided weakness and 
left optic atrophy. She was put on dexamethasone, phenytoin and mannitol. She was observed for 15 days when she became disoriented and had spontaneous rupture of membranes. It was decided to take her up for neurosurgery immediately after delivering the baby. An emergency cesarean section was done under general anaesthesia with rapid sequence induction and delivered a $2 \mathrm{~kg}$ healthy baby. Patient was shifted to the neurosurgery operation theater at the adjacent super-specialty hospital and a near total excision of meningioma was done by the neurosurgeon. On follow up after 5 months, patient is doing better with slight residual weakness in the right side with loss of vision in the left eye with a healthy breastfed baby.

\section{Discussion}

Pregnancy with meningioma or intracranial space occupying lesion in general has been discussed since the first case was reported in the late $19^{\text {th }}$ century [1]. In 1938 first case of meningioma with pregnancy was reported by Cushing and Eisenhardt. Brain tumors occur with the same frequency in age matched pregnant and non-pregnant woman [2]. Glioma is the most common followed by meningioma in $28 \%$ of the cases [3]. Meningiomas are mostly silent brain tumors with slow growth. However they may get detected during pregnancy due to increase in size secondary to either water retention, enhanced vascularity or progesterones. The pathophysiology is debatable. Most of the meningiomas express progesterone receptors but not estrogen receptors [4-7]. Meningiomas mostly become symptomatic in pregnancy and luteal phase of menstrual cycle. Thence, both fluid retention and progesterones are possible etiologies.

In the cases described, the concerns were diagnosis of meningioma, drugs in pregnancy, timing of termination of pregnancy or neurosurgery and the type of anaesthesia for the same. Pregnancy with headache and vomiting might be misdiagnosed as hyperemesis gravidarum and seizures could present like that in eclampsia [8].
However, visual impairment, focal seizures and lateralizing neurological deficits like hemiparesis should prompt the diagnosis of intracranial lesion. Diagnosis is best achieved with MRI or CT scan. But the iodinated contrast though inert, can cause dehydration and hence impair fetal supply. Adequate hydration is to be maintained to avoid this complication [9]. Gadolinium contrast crosses the placenta but there is no evidence of teratogenicity. However, its use is not validated [3].

The use of corticosteroids like dexamethasone is useful to control cerebral edema and promote fetal lung maturity. However there is risk of fetal hypo-adrenalism in prolonged use $[8,10]$. Mannitol should be used cautiously during pregnancy due the risk of compromise in fetal circulation. In doses $0.5-1 \mathrm{~g} / \mathrm{kg}$, it is considered safe [11]. Anti-epileptics are a must in managing these cases, as seizures are associated with major maternal and fetal complications which are more than the risk of antiepileptic drugs $[8,10]$. In our cases, both were in their third trimester and thus teratogenicity with anti-epileptics was not a concern.

Antepartum excision of meningioma is indicated in cases with progressive neurological deterioration, impending herniation or malignant tumors $[8,12]$. The ongoing pregnancy complicates the tumor by predisposing to edema formation due to increased nitric oxide production and increased perfusion [12]. On the other hand, neurosurgical intervention puts the fetus under risk as anaesthesia and surgery can result in fetal hypoxemia, trigger preterm labour and thus the complications of prematurity [13]. Smooth rapid sequence induction of anaesthesia, fluid maintenance and blood pressure monitoring with or without tocolytics are advised $[12,13]$.

If the neurological condition is stable like that in the first case, brain surgery can wait until or after delivery of the baby. However, in a stable patient with signs of brain edema as in second case, 
fetal lung maturity is accelerated and craniotomy is preferably done with the delivery [14]. Normal labour is not contraindicated with intracranial neoplasia $[15,16]$. But sufficient pain relief is a must to avoid the risks of raised intracranial pressures. Either for normal labour or cesarean section, epidural aneasthesia can be used but there can be serious complications in case there is dural puncture or wet tap $[7,15]$. If neurosurgery is planned with the delivery, elective cesarean section under general anaesthesia is preferable. The mode of delivery is tailored to the clinical situation and obstetrician's preferences.

\section{Conclusion}

Thus a multimodality approach with the aid of management strategies in the literature, guide the successful outcome of a case of meningioma in pregnancy.

Contributors: AKR: manuscript writing, patient management, surgery; PS: manuscript editing, patient management, surgery; GG, AR: critical inputs into the manuscript, patient management. AKR will act as guarantor. All authors approved the final version of the manuscript.

Funding: None; Competing interests: None stated.

\section{References}

1. Bernard MH. Sarcome cerebral a' evolution rapide au cours de la grossesse et pendant les suites des couches. Bull Soc d'Obst de Paris. 1898;1:296-298.

2. Roelvink NC, Kamphorst W, van Alphen HA, Rao BR. Pregnancy-related primary brain and spinal tumors. Arch Neurol. 1987;44:209-215.

3. Stevenson CB, Thompson RC. The clinical management of intracranial neoplasms in pregnancy. Clin Obstet Gynecol. 2005;48:24-37.

4. Donnell MS, Meyer GA, Donegan WL. Estrogen- receptor protein in intracranial meningiomas. J Neurosurg. 1979;50:499-502.

5. Cahill DW, Bashirelahi N, Solomon LWE, Dalton T, Saloman M, Tucker TB. Estrogen and progesterone receptors in meningiomas in relation to clinical and pathologic features. J Neurosurg. 1984;60:985-993.

6. Glick RP, Molteni A, Fors EM. Hormone binding in brain tumors. Neurosurgery. 1983;13:513-519.

7. Ravindra VM, Braca JA, Jensen RL, Duckworth EAM. Management of intracranial pathology during pregnancy: Case example and review of management strategies. Surg Neurol Int. 2015;6:43.

8. Kanaan I, Jallu A, Kanaan H. Management strategy for meningioma in pregnancy: A clinical study. Skull Base. 2003;13:197-203.

9. Dalessio DJ. Neurologic diseases. In: Burrow GN, Ferris TF, eds. Medical complications during pregnancy. Philadelphia: WB Saunders. 1982:435-447.

10. Isla A, Alvarez F, Gonzalez A, Garcia-Grande A, PerezAlvarez M, Garcia-Blazquez M. Brain tumor and pregnancy. Obstet Gynaecol. 1997;89:19-22.

11. Tuncali B, Aksun M, Katircioglu K, Akkol I, Savaci S. Intraoperative fetal heart rate monitoring during emergency neurosurgery in a parturient. J Anaesth. 2006;20:40-43.

12. Sahu S, Lata I, Gupta D. Management of pregnant female with meningioma for craniotomy. J Neurosci Rural Pract. 2010;1:35-37.

13. Marulasiddappa V, Raghavendra BS, Nethra HN. Anaesthetic management of a pregnant patient with intracranial space occupying lesion for craniotomy. Indian J Anaesth. 2014;58:739-741.

14. Lynch JC,Gouvêa F, Emmerich JC, et al. Management strategy for brain tumor diagnosed during pregnancy. British Journal of Neurosurgery. 2011;25:225-230.

15. Finfer SR. Management of labour and delivery in patients with intracranial neoplasms. British Journal of Anaesthesia. 1991;67:784-787.

16. Ng J, Kitchen N. Neurosurgery and pregnancy. J Neurol Neurosurg Psychiatry. 2008;79:745-752. 